\title{
Influência do consumo alimentar e do padrão de atividade física sobre o estado nutricional de adolescentes de Piedade, São Paulo
}

\author{
Influence of food intake and physical activity patterns on the nutritional status of adolescents from \\ Piedade, São Paulo, Brazil
}

Carla Cristina Enes ${ }^{1}$, Giovana Eliza Pegolo², Marina Vieira da Silva ${ }^{3}$

\section{RESUMO}

Objetivo: Identificar os fatores dietéticos e de atividade física associados ao estado nutricional de adolescentes escolares.

Métodos: Estudo transversal com 105 adolescentes de ambos os sexos, idade de 10 a 14 anos, matriculados em escolas públicas de Piedade (SP). Foram coletadas informações sobre consumo alimentar (recordatório 24 horas), padrão de atividade física (atividades físicas e sedentárias) e estado nutricional avaliado pelo índice de massa corporal. Consideraram-se insuficientemente ativos os adolescentes que praticavam menos de 300 minutos/semana de atividade física. Foram classificados como sedentários aqueles que dedicavam período de tempo igual ou superior a duas horas por dia a atividades passivas. Para identificar os fatores associados ao estado nutricional, recorreu-se à regressão linear múltipla.

Resultados: A prevalência de excesso de peso foi maior entre os meninos $(17,9$ versus $13,6 \%, p<0,05)$. Dos adolescentes, $18 \%$ foram considerados insuficientemente ativos. Não houve associação entre as variáveis dietéticas e o estado nutricional. Observou-se diferença significativa apenas para o tempo dedicado para jogar videogame $(p=0,005)$, que foi maior nos adolescentes eutróficos. Sete em cada dez adolescentes dedicavam mais de duas horas diárias às atividades sedentárias, como assistir à televisão, usar o computador e jogar videogame. $\mathrm{Na}$ análise múltipla, somente a idade se associou positivamente ao IMC $(p=0,001)$.

Instituição: Escola Superior de Agricultura "Luiz de Queiroz" da Universidade de São Paulo (ESALQ/USP), Piracicaba, SP, Brasil

'Doutoranda em Saúde Pública pela Faculdade de Saúde Pública da USP, São Paulo, SP, Brasil

2Doutoranda em Alimentos e Nutrição da Faculdade de Ciências Farmacêuticas da Universidade Estadual de São Paulo "Júlio de Mesquita Filho", Araraquara, SP, Brasil

${ }^{3}$ Professora Doutora do Departamento de Agroindústria, Alimentos e Nutrição da ESALQ/USP, Piracicaba, SP, Brasil
Conclusões: Há necessidade de novas investigações de caráter prospectivo que estabeleçam relações mais claras entre os fatores relacionados ao estilo de vida e o índice de massa corporal em adolescentes.

Palavras-chave: adolescente; atividade física; consumo de alimentos; estado nutricional.

\section{ABSTRACT}

Objective: To identify dietary and physical activity factors associated to the nutritional status of adolescent students.

Methods: Cross-sectional study of 105 adolescents of both genders, aged 10 to 14 years, studying at public schools in Piedade, São Paulo, Brazil. Data regarding food intake (24 hour recall), physical activity patterns (physical and sedentary activities) and nutritional status measured by body mass index were collected. The adolescents were characterized as insufficiently active if physical activities were practiced for less than 300 minutes per week. They were considered sedentary if they spent two hours or more per day in passive activities. Multiple linear regression was performed to identify factors associated to the nutritional status.

Results: The prevalence of overweight was higher among boys $(17.9$ versus $13.6 \%, p<0.05)$ and $18.0 \%$ of the adolescents were considered insufficiently active. There was no association between dietary and nutritional status variables. A significant difference was observed only for the time spent

\author{
Endereço para correspondência: \\ Carla Cristina Enes \\ Rua Capitão Alfredo Cardoso, 54 - Jardim Faculdade \\ CEP 18030-310 - Sorocaba/SP \\ E-mail: cenes@usp.br
}

Fonte financiadora: $\mathrm{O}$ trabalho integra a pesquisa Contrastes regionais nos custos, qualidade e operacionalização do Programa Nacional de Alimentação Escolar (PNAE) e seu impacto sobre os padrões alimentares da população brasileira, financiada pelo Conselho Nacional de Desenvolvimento Científico (CNPq), Processo n 504369/2003-2.

Recebido em: 29/10/08

Aprovado em: 26/3/09 
playing videogames $(p=0.005)$, that was higher among eutrophic adolescents. Seven in ten adolescents spent more than two hours a day on sedentary activities (watching television, using the computer and playing videogames). In multiple regression analysis, only age was positively associated with the BMI $(p=0.001)$.

Conclusions: Further prospective investigations are necessary to establish clearer relationships among factors related to lifestyle and body mass index among adolescents.

Key-words: adolescent; motor activity; food consumption; nutritional status.

\section{Introdução}

O aumento da prevalência de obesidade na população adulta observado nos últimos anos tem sido também constatado em idades cada vez mais precoces. Assim, o problema de excesso de peso entre os jovens passou a ocupar papel de destaque nas discussões relacionadas à saúde dessa população ${ }^{(1)}$. O estado nutricional do adolescente é de particular interesse, pois a presença de obesidade nesta faixa etária tem relação com o aparecimento de doenças crônicas não transmissíveis, distúrbios na esfera emocional, além de comprometer a postura e causar alterações no aparelho locomotor $^{(2)}$. A ocorrência de obesidade em idades precoces também pode acarretar prejuízos à saúde em longo prazo, com aumento do risco de mortalidade em adultos que foram obesos durante a infância e adolescência ${ }^{(3)}$.

Embora seja consenso ser a obesidade uma doença complexa e de etiologia multifatorial, fatores ambientais frequentemente se relacionam ao seu aparecimento. As mudanças ocorridas nos padrões alimentares nas últimas décadas, como o aumento do consumo de açúcares simples, alimentos industrializados e ingestão insuficiente de frutas e hortaliças, contribuem diretamente para o ganho de peso nesse grupo populacional ${ }^{(4)}$. Além disso, a redução progressiva da prática de atividade física associada ao maior tempo dedicado às atividades de baixa intensidade, como assistir televisão, usar computador e jogar videogame, exercem papel no ganho de peso dos adolescentes ${ }^{(5,6)}$. Evidências confirmam que a prática de atividade física dos jovens se relaciona de maneira inversa com o risco de doenças crônicas não transmissíveis, como a obesidade, além de exercer um efeito positivo na qualidade de vida. A importância da manutenção de uma vida ativa também se justifica pelo fato de os padrões de atividade na adolescência determinarem parte dos níveis de atividade física na idade adulta ${ }^{(7)}$.
Embora muitos estudos tenham sido realizados com vistas à identificação dos determinantes do sobrepeso e da obesidade durante a adolescência, a relação entre o consumo alimentar, o padrão de atividade e o estado nutricional ainda é controversa. O objetivo do presente estudo foi identificar os fatores dietéticos e de atividade física associados ao estado nutricional de adolescentes.

\section{Métodos}

Trata-se de um estudo transversal do qual participaram 105 adolescentes de ambos os sexos, com idade entre 10 e 14 anos, matriculados em escolas públicas e residentes, em sua maioria, em áreas rurais do município de Piedade (SP).

De acordo com o Censo Demográfico de 2000, 56\% da população desse município residia na zona rural ${ }^{(8)}$. Segundo dados do Censo Escolar ${ }^{(9)}$, o município contava, em 2004, com 8.035 alunos regularmente matriculados nas redes estaduais e municipais de ensino, sendo 4.096 da primeira à quarta série e 3.939 da quinta à oitava série. Para o cálculo do tamanho da amostra, as escolas foram sorteadas de maneira que a probabilidade de uma unidade ser incluída na amostra fosse proporcional ao número de alunos. Para isso, foi elaborada uma tabela com o nome das escolas do município, efetuando-se a soma (acumulada) do número de alunos, de maneira que cada unidade de ensino ficasse associada a um intervalo de valores de frequência acumulada de escolares. Se os valores inicial e final do intervalo para a h-ésima escola são $n_{h i}$ e $n_{h f}$, respectivamente, então o número de alunos dessa escola $\mathrm{N}_{\mathrm{h}}=\mathrm{n}_{\mathrm{hf}}-\mathrm{n}_{\mathrm{hi}}+1$, sendo $\mathrm{N}$ o número de alunos de todas as escolas consideradas no município. Com o auxílio de um software, foi gerado um número aleatório inteiro de 1 a $\mathrm{N}$, todos com igual probabilidade. Em seguida, verificou-se qual era a escola cujo intervalo de frequência acumulada incluía esse número inteiro obtido, passando essa escola a integrar a amostra. Novos números foram gerados até que se completasse o número de escolas previamente estabelecido $(n=6)$.

$\mathrm{Na}$ segunda etapa, foram sorteados de forma aleatória simples seis alunos de cada classe existente na escola, visando a assegurar, ao final do processo, o total de três alunos de cada classe. O sorteio foi realizado em sala de aula com o auxílio da lista de frequência adotada pelos professores. Após a realização dos procedimentos de amostragem, foram sorteados 150 escolares. Ressalta-se que, para este estudo, a amostra final foi constituída por 105 alunos, devido à exclusão daqueles com idade inferior a dez anos $(n=45)$, já que se optou por trabalhar apenas com a população de adolescentes. 
Os adolescentes foram entrevistados em maio de 2005 por pesquisadores treinados, nas próprias instituições de ensino. A totalidade dos participantes devolveu o termo de consentimento livre e esclarecido assinado pelos pais ou responsáveis. O estudo foi aprovado pelo Comitê de Ética da Faculdade de Odontologia de Piracicaba da Unicamp.

As variáveis investigadas, além das demográficas, relativas ao sexo e à idade dos participantes, foram:

- Consumo alimentar: avaliado por meio da aplicação de um Recordatório de 24 horas, utilizando-se material fotográfico com diferentes utensílios como pratos, copos e talheres para garantir a maior fidedignidade das informações. Para os cálculos nutricionais, utilizou-se o software Nutwin versão $1.5^{(10)}$. Foram analisados os dados referentes à ingestão total de energia diária, participação dos lipídeos na dieta e ingestão diária de fibras.

- Atividade física: a Atividade Física Habitual (AFH) foi avaliada por meio de um questionário elaborado por Florindo $e t a l^{(11)}$. O nível de atividade física dos adolescentes foi analisado a partir de informações referentes à prática de atividades de lazer, incluindo a educação física escolar, a prática de exercício físico estruturado (academias, treinamento em esportes, clubes) e a forma de deslocamento ativo (caminhar ou pedalar) para a escola. Optou-se por excluir dos cálculos a educação física escolar, já que esta não contribuiu de forma expressiva para discriminar o nível de atividade física dos adolescentes. Os adolescentes foram classificados em 'insuficientemente ativos' e 'ativos', com base no ponto de corte de 300 minutos semanais estabelecido pelo United Kingdom Expert Consensus Group ${ }^{(12)}$.

- Atividades sedentárias: avaliou-se o tempo gasto diariamente (em horas) assistindo televisão, jogando videogame ou usando computador. Os adolescentes foram classificados em duas categorias, conforme recomendação da American Academy of Pediatrics ${ }^{(13)}: \leq 2$ horas/dia (não sedentários) e $>2$ horas/dia (sedentários).

- Estado nutricional: a partir das medidas de peso e altura foi calculado o índice de massa corporal (IMC). Com base nos valores de IMC, os adolescentes foram classificados em quatro categorias quanto ao estado nutricional, de acordo com a proposta pela Organização Mundial da Saúde (OMS) ${ }^{(14)}$, segundo sexo e idade: baixo peso $(<\mathrm{P} 3)$, eutrófico $(\geq \mathrm{P} 3 \mathrm{e}<\mathrm{P} 85)$, sobrepeso $(\geq \mathrm{P} 85$ e $<$ P97) e obeso $(\geq$ P97). Diante da baixa ocorrência de adolescentes com baixo peso $(8,6 \%)$ e obesidade $(2,9 \%)$, realizou-se uma nova classificação para fins de análise: eutrófico (baixo peso + eutrófico) e excesso de peso (sobrepeso + obeso).

$\mathrm{Na}$ análise estatística, realizou-se o teste de KolmogorovSmirnov para avaliar a aderência dos dados à distribuição normal. Aplicou-se o teste $t$ de Student com o objetivo de comparar o consumo alimentar e o padrão de atividade física de acordo com o sexo e categorias de estado nutricional. Para comparar a prevalência de excesso de peso segundo o sexo, utilizou-se teste do qui-quadrado. Um modelo de regressão linear múltiplo foi estimado com o objetivo de analisar os fatores associados ao estado nutricional dos adolescentes. A estratégia de modelagem adotada foi "stepwise forward", partindo-se do modelo mais simples para o mais complexo. As variáveis que apresentaram valor de $p<0,20$ na análise univariada entraram no modelo, seguindo a ordem do valor de $p$ de maior significância para a menor. Permaneceram no modelo final, além das variáveis de controle, somente aquelas que apresentaram significância estatística ou ajustaram em pelo menos $10 \%$ os coeficientes de regressão $\left(\beta_{i}\right)$ das variáveis que já estavam no modelo. Adotou-se o nível de significância de 5\%. As análises foram realizadas com o pacote estatístico SPSS versão 11.5 for Windows.

\section{Resultados}

Participaram do estudo 105 adolescentes com idade média de $12,4 \pm 1,3$ anos, sendo $63 \%$ do sexo feminino. A média de idade das meninas foi de $12,4 \pm 1,4$ e dos meninos de $12,5 \pm 1,3$ anos $(p>0,05)$.

A prevalência de excesso de peso foi de 15,2\%. Embora não tenha sido observada diferença significativa para os valores médios de IMC segundo o sexo (Tabela 1), a prevalência de excesso de peso foi maior entre os meninos (17,9 versus $13,6 \%, p<0,05)$. Em relação às variáveis dietéticas, os resultados mostram não haver diferença significante entre os grupos de acordo com o sexo (Tabela 1).

O tempo médio dedicado à prática de atividades físicas foi de cerca de três horas por dia (1.218 minutos/semana) para os meninos e de duas horas por dia (882 minuto/ semana) para as meninas. Essa diferença apresentou valor de $p$ limítrofe para significância $(p=0,05)$ (Tabela 1$)$. Dos adolescentes, $18,0 \%$ foram considerados insuficientemente ativos (<300 min/semana) e $14,3 \%$ não praticavam nenhuma atividade. Os esportes mais praticados pelos adolescentes foram: futebol $(45,6 \%)$, vôlei $(14,4 \%)$ e futsal $(12,2 \%)$, sendo que outras atividades também foram 
citadas com menor frequência. Verificou-se que $73,3 \%$ dos adolescentes dedicavam mais de duas horas diárias às atividades sedentárias como assistir à televisão, usar o computador e jogar videogame. Desse total, $64 \%$ eram do sexo feminino. Conforme apresentado na Tabela 1 , o tempo médio (em h/dia) gasto assistindo TV $(p=0,03)$ e jogando videogame $(p=0,04)$ apresentou diferença estatística entre meninos e meninas.

$\mathrm{Na}$ Tabela 2, que compara adolescentes obesos e eutróficos, observa-se que a variável idade apresentou valor de $p$ limítrofe para significância $(p=0,05)$, sendo a média de idade maior entre os adolescentes com excesso de peso. Embora não tenha sido observada diferença significativa entre os eutróficos e aqueles com excesso de peso para as variáveis dietéticas analisadas, verificou-se que, entre os adolescentes com excesso de peso, a ingestão de energia e de fibras foi menor e a participação dos lipídeos na dieta foi ligeiramente superior. Não foram identificadas diferenças significativas para a prática de atividades físicas entre os adolescentes eutróficos e com excesso de peso (Tabela 2). Em relação às atividades sedentárias, observou-se diferença estatisticamente significativa apenas para o tempo dedicado para jogar videogame $(p=0,005)$, que foi maior entre os adolescentes eutróficos.

$\mathrm{Na}$ Tabela 3 estão apresentados os resultados da análise de regressão linear múltipla realizada com o intuito de

Tabela 1 - Características da população de estudo de acordo com o sexo

\begin{tabular}{lccc}
\hline Variável & $\begin{array}{c}\text { Meninos } \\
(\mathbf{n = 3 9 )}\end{array}$ & $\begin{array}{c}\text { Meninas } \\
(\mathbf{n = 6 6 )}\end{array}$ & Valor de $\boldsymbol{p}$ \\
\hline IMC $\left(\mathrm{kg} / \mathrm{m}^{2}\right)$ & $18,5 \pm 2,9$ & $18,3 \pm 2,7$ & 0,78 \\
Ingestão de energia (kcal/dia) & $1913 \pm 749$ & $1873 \pm 694$ & 0,37 \\
Lipídeos da dieta (\%) & $29,7 \pm 8,0$ & $28,3 \pm 6,6$ & 0,56 \\
Fibras (g/dia) & $18,5 \pm 10,0$ & $17,4 \pm 8,2$ & 0,64 \\
Atividade física (min/semana) & $1218 \pm 899$ & $882 \pm 899$ & 0,05 \\
Atividades sedentárias (h/dia) & & & \\
Assistir TV & $3,3 \pm 2,5$ & $4,4 \pm 3,0$ & 0,03 \\
Jogar videogame & $0,4 \pm 0,9$ & $0,1 \pm 0,4$ & 0,04 \\
$\quad$ Usar computador & $0,1 \pm 0,2$ & $0,1 \pm 0,2$ & 0,37 \\
\hline
\end{tabular}

IMC: índice de massa corporal; dp: desvio padrão

Tabela 2 - Estado nutricional dos adolescentes segundo variáveis demográficas, dietéticas e de padrão de atividade física

\begin{tabular}{lccc}
\hline & \multicolumn{2}{c}{ Classificação do estado nutricional } & \\
\cline { 2 - 3 } Variável & $\begin{array}{c}\text { Eutrófico } \\
(\mathbf{n}=\mathbf{8 9})\end{array}$ & $\begin{array}{c}\text { Excesso de peso } \\
(\mathbf{n}=\mathbf{1 6})\end{array}$ & Valor de $\boldsymbol{p}$ \\
\hline Idade (anos) & $12,3 \pm 1,4$ & $13,0 \pm 1,0$ & 0,05 \\
Ingestão de energia (kcal/dia) & $1937 \pm 672$ & $1616 \pm 878$ & 0,18 \\
Lipídeos da dieta (\%) & $28,6 \pm 7,0$ & $29,9 \pm 8,1$ & 0,57 \\
Fibras (g/dia) & $18,0 \pm 8,8$ & $16,9 \pm 9,6$ & 0,68 \\
Atividade física (min/semana) & $1050 \pm 857$ & $924 \pm 1197$ & 0,72 \\
Atividades sedentárias (h/dia) & & & \\
Assistir TV & $3,9 \pm 2,8$ & $4,3 \pm 2,9$ & 0,63 \\
Jogar videogame & $0,3 \pm 0,7$ & $0,04 \pm 0,1$ & 0,005 \\
Usar computador & $0,1 \pm 0,2$ & $0,04 \pm 0,1$ & 0,39 \\
\hline
\end{tabular}


identificar os fatores associados ao índice de massa corporal dos adolescentes. Somente a variável idade se manteve significativamente associada ao IMC dos adolescentes após a análise múltipla. Observa-se que essa associação é positiva, ou seja, com o aumento da idade ocorre um aumento no IMC dos adolescentes. Assim, para cada ano adicional, há um aumento de $0,656 \mathrm{~kg} / \mathrm{m}^{2}$ no IMC. Esse achado confirma a influência da idade sobre o IMC independentemente do tempo gasto jogando videogame e assistindo à TV. A variável “jogar videogame” perdeu sua significância estatística ao ser analisada simultaneamente às demais variáveis de interesse; no entanto, foi mantida no modelo, assim como "assistir TV", como variável de ajuste.

\section{Discussão}

A prevalência de excesso de peso e de sedentarismo encontrada neste estudo foi menor do que a observada em pesquisas realizadas com populações de idade semelhante ${ }^{(15-}$ 17). Uma possível explicação para essa prevalência menor é o fato de os escolares residirem em um município onde a principal atividade econômica se baseia na agricultura, sendo comum que os adolescentes, especialmente do sexo masculino, auxiliem nas atividades agrícolas da família (atividades não avaliadas neste estudo). Resultados obtidos por Pontin ${ }^{(18)}$, a partir de um estudo com escolares de um município com atividades predominantemente agrícolas, também apontam reduzida prevalência de excesso de peso (3,1\% para meninos e $2,2 \%$ para meninas).

A proporção de adolescentes classificados como insuficientemente ativos foi inferior àquela identificada por outros estudos realizados no Brasil. Pierine $e t$ al $^{(19)}$, ao avaliarem o nível de atividade física de 441 adolescentes escolares em Botucatu (SP), verificaram que $54 \%$ eram insuficientemente ativos. Maitino ${ }^{(20)}$ constatou que a inatividade física esteve presente em $42 \%$ dos escolares estudados.

A maior prevalência de excesso de peso observada em adolescentes do sexo masculino corrobora os achados de Silva $e$ e $a^{(21)}$, que avaliaram 5.028 adolescentes com idade entre 15 e 19 anos. Os adolescentes do sexo masculino gastam, em média, maior tempo praticando atividades físicas. Outros estudos também observaram relação entre hábitos sedentários e sexo, sendo os meninos mais ativos fisicamente ${ }^{(16,21,22)}$.

O padrão de sedentarismo encontrado entre os adolescentes deste estudo corrobora os achados de Silva et al ${ }^{(21)}$, que verificaram que cerca de $70 \%$ dos adolescentes dedicavam tempo igual ou superior a duas horas diárias em atividades passivas. É importante destacar que as recomendações da Academia Americana de Pediatria ${ }^{(23)}$, com base nas evidências que comprovam a relação entre sedentarismo e ganho de peso, sugerem que o tempo dedicado a atividades de baixa intensidade, especialmente assistir TV, seja limitado a uma ou duas horas diárias. Na presente investigação, na qual os hábitos sedentários foram analisados separadamente, os resultados revelam que o tempo médio assistindo à televisão é maior entre as meninas, ao passo que os meninos gastam mais tempo jogando videogame. Esses resultados poderiam ser previstos, tendo em vista a preferência dos meninos por jogos eletrônicos.

A idade se revelou positivamente associada ao índice de massa corporal dos adolescentes. Esse resultado já era esperado, uma vez que, no período da adolescência, sobretudo em sua fase inicial, ocorrem importantes mudanças na composição corporal. Tais mudanças, na maioria das vezes, vêm acompanhadas de elevação de peso maior que a de estatura, levando, portanto, ao aumento do IMC.

As análises dietéticas revelam que o consumo alimentar atual em adolescentes eutróficos e com excesso de peso é muito semelhante. $\mathrm{O}$ menor consumo de energia observado nos adolescentes com excesso de peso pode ser justificado pela subestimação da ingestão alimentar por esse grupo, conforme descrito por Patrick et al ${ }^{(24)}$ ou mesmo devido à ocorrência de causalidade reversa: adolescentes com excesso de peso podem ter diminuído o consumo com o objetivo de perder peso. A ingestão de fibras foi discretamente superior em adolescentes eutróficos, porém sem significância estatística. Lima et al ${ }^{(25)}$, em estudo transversal realizado

Tabela 3 - Variáveis independentes associadas ao índice de massa corporal dos adolescentes

\begin{tabular}{lcccc}
\hline & $\beta$ & IC95\% $(\beta)$ & Valor de $\boldsymbol{p}$ & $\mathbf{r}^{2}$ ajustado \\
\hline Idade (anos) & 0,656 & $0,266-1,045$ & 0,001 & 0,086 \\
Jogar videogame (h/dia) & $-0,352$ & $-1,562$ & 0,374 & 0,084 \\
Assistir TV (h/dia) & 0,039 & $-0,377$ & 0,684 & 0,077 \\
\hline
\end{tabular}

IC: intervalo de confiança 
com crianças e adolescentes, encontraram correlação negativa entre o consumo de fibras e o IMC para o grupo de meninas obesas. A porcentagem de calorias provenientes dos lipídeos também não diferiu segundo o estado nutricional, resultado que corrobora os achados de Patrick et al ${ }^{(24)}$. No entanto, observa-se que o consumo de lipídeos é ligeiramente superior entre os adolescentes com excesso de peso. Destaca-se, ainda, que a contribuição média dos lipídeos na dieta se revelou muito próxima do limite máximo aceitável (30\%).

Embora seja consenso que o crescimento da prevalência de sobrepeso e obesidade seja decorrente do aumento no consumo de alimentos com elevada densidade energética e, especialmente, ricos em lipídeos e carboidratos simples, esse fato isoladamente não é capaz de explicar o aumento exponencial de obesidade no mundo. Também, apesar das evidências que relacionam a prática de atividade física ao peso corporal, apenas 16 em 31 estudos com crianças e seis em 21 realizados com adolescentes demonstraram uma associação significativa entre essas variáveis ${ }^{(26)}$. $\mathrm{Na}$ presente pesquisa, não foi possível verificar relação entre o tempo gasto com atividades físicas e o estado nutricional dos adolescentes.

Por outro lado, hábitos sedentários têm se revelado frequentemente fatores de risco para a ocorrência de excesso de peso ${ }^{(17,27,28)}$. Dentre as atividades sedentárias mais relacionadas ao aumento de peso corporal está o hábito de assistir à televisão, que se deve à influência que esse meio de comunicação exerce sobre as escolhas alimentares. Além disso, estudos revelam que o hábito de assistir à televisão leva ao maior consumo de alimentos ricos em monossacarídeos e lipídeos e redução do consumo de frutas e hortaliças ${ }^{(29)}$. Wong et al ${ }^{(30)}$, em 1992, já chamavam a atenção para o tempo excessivo dedicado a essa atividade como um sinal mundial para identificar crianças e adolescentes inseridos em estilos de vida que valorizam hábitos inadequados. Baruki et $a l^{(7)}$ e Silva et a $l^{(21)}$ obtiveram resultados que apoiam a existência de associação entre o estado nutricional e hábitos sedentários. Os autores constataram que crianças eutróficas são mais ativas e passam menos tempo em atividades sedentárias em comparação àquelas com excesso de peso. No presente estudo, identificou-se associação inversa entre o tempo gasto jogando videogame e o estado nutricional dos adolescentes na análise univariada, corroborando os achados de Hallal et $a l^{(17)}$, mas essa associação não permaneceu ao se controlar a idade dos paciente. Uma possível explicação para esse achado é que grande parte dos jogos eletrônicos se baseia em lutas ou modalidades esportivas, podendo servir de estímulo à prática de atividade física entre os jovens. Outra possibilidade seria a ocorrência de causalidade reversa ou viés de informação, pois os adolescentes com excesso de peso podem ter subestimado o tempo gasto com essa atividade. Vale lembrar que o pequeno número de casos de adolescentes com excesso de peso pode ter comprometido a demonstração das associações.

É importante mencionar também que o IMC pode ser considerado um fraco estimador da gordura corporal em adolescentes, particularmente quando o objetivo é associá-lo ao nível de atividade física. Isto se deve ao fato de as mudanças na composição corporal decorrentes da maturação sexual não serem captadas pelo IMC ${ }^{(31)}$.

Algumas limitações deste estudo devem ser consideradas. A natureza transversal dos dados pode ter contribuído para a ocorrência de causalidade reversa na associação entre jogar videogame e estado nutricional. O tamanho da amostra analisada também pode ter comprometido o poder estatístico dos testes, bem como a confirmação da hipótese inicial do estudo, o que poderia explicar o fato de somente a idade ter apresentado associação com o IMC na análise múltipla. Em última análise, é preciso levar em conta as dificuldades de avaliar a associação entre o padrão alimentar e atividade física com o estado nutricional de adolescentes, já que esse grupo se encontra em uma fase de definição de padrões de estilo de vida.

Os resultados obtidos na presente investigação revelam que, das sete variáveis analisadas, apenas a idade se associou ao índice de massa corporal dos adolescentes. É possível que o tamanho da amostra e a natureza transversal dos dados possam ter contribuído para a não ocorrência de associação entre as variáveis de interesse. Embora os estudos de natureza transversal não permitam inferir causalidade, são importantes para gerar hipóteses e direcionar a realização de estudos prospectivos. Portanto, são necessárias novas investigações de caráter prospectivo para estabelecer relações mais claras entre os fatores relacionados ao estilo de vida e o índice de massa corporal em adolescentes. 


\section{Referências bibliográficas}

1. Klein $\mathrm{S}$, Wadden $\mathrm{T}$, Sugerman HJ. AGA technical review on obesity. Gastroenterology 2002;123:882-932.

2. Dietz WH. Health consequences of obesity in youth: childhood predictors of adult disease. Pediatrics 1998;101:518-25.

3. Must A. Morbidity and mortality associated with elevated body weight in children and adolescents. Am J Clin Nutr 1996 (Suppl 3);63:445-7.

4. Toral N, Slater B, Silva MV. Consumo alimentar e excesso de peso de adolescentes de Piracicaba, São Paulo. Rev Nutr 2007;20:449-59.

5. Berkey CS, Rockett HR, Field AE, Gillman MW, Frazier AL, Camargo CA et al. Activity, dietary intake, and weight changes in a longitudinal study of preadolescent and adolescent boys and girls. Pediatrics 2000;105:E56.

6. Ronque ER, Cyrino ES, Dórea VR, Helio Junior S, Galdi EH, Arruda M. Prevalência de sobrepeso e obesidade em escolares de alto nível socioeconômico em Londrina, Paraná, Brasil. Rev Nutr 2005;18:709-17.

7. Baruki SB, Rosado LE, Rosado GP, Ribeiro RC. Associação entre estado nutricional e atividade física em escolares da Rede Municipal de Ensino em Corumbá, MS. Rev Bras Med Esporte 2006;12:90-4.

8. Instituto Brasileiro de Geografia e Estatística [homepage on the Internet]. Censo 2000. [cited 2005 Jul 9]. Available from: http://www.ibge.gov.br

9. Brasil. Ministério da Educação. Instituto de Pesquisas Educacionais Anísio Teixeira - INEP [homepage on the internet]. Resultados finais do Censo Escolar de 2004 [cited 2005 Jul 9]. Available from: http://www.inep.gov.br/ basica/censo/

10. Anção MS, Cuppari L, Draibe S, Sigulem D. Informática em terapia nutricional. In: Magnoni D, Cukier, editores. Perguntas e respostas em nutrição clínica. $1^{\text {a }}$ ed. São Paulo: Roca; 2001. p. 361-4.

11. Florindo AA, Romero A, Peres SV, Silva MV, Slater B. Development and validation of a physical activity assessment questionnaire for adolescents. Rev Saude Publica 2006;40:802-9.

12. Pate RR, Freedson PS, Sallis JF, Taylor WC, Sirard J, Trost SG et al. Compliance with physical activity guidelines: prevalence in a population of children and youth. Ann Epidemiol 2002;12:303-8.

13. American Academy of Pediatrics. Television and the family. Elk Grove Village (IL): American Academy of Pediatrics; 1986.

14. de Onis M, Onyango AW, Borghi E, Siyam A, Nishida C, Siekmann J. Development of a WHO growth reference for school-aged children and adolescents. Bull World Health Organ 2007;85:660-7.

15. Oehlschlaeger MH, Pinheiro RT, Horta B, Gelatti C, San'Tana P. Prevalence of sedentarism and its associated factors among urban adolescents. Rev Saude Publica 2004;38:157-63.

16. Frutuoso MF, Bismarck-Nasr EM, Gambardella AM. Energy expenditure reduc- tion and overweight in adolescents. Rev Nutr 2003;16:257-63.

17. Hallal PC, Bertoldi AD, Gonçalves H, Victora CG. Prevalence of sedentary lifestyle and associated factors in adolescents 10 to 12 years of age. Cad Saude Publica 2006;22:1277-87.

18. Pontin N. Prevalência de obesidade em adolescentes na faixa etária de 15 a 19 anos, nas escolas do ensino médio do município de Ouro, SC, Brasil [dissertação de mestrado]. Joaçaba (SC): Universidade do Oeste de Santa Catarina; 2005.

19. Pierine DT, Carrascosa AP, Fornazari AC, Watanabe MT, Catalani MC, Fukuju MM et al. Composição corporal, atividade física e consumo alimentar de alunos do ensino fundamental e médio. Motriz 2006;12:113-24.

20. Maitino EM. Aspectos de risco coronariano em casuística de crianças de escola pública de primeiro grau em Bauru, SP. Rev Brasil At Fis Saude 1997;2:37-52.

21. Silva KS, Nahas MV, Hoefelmann LP, Lopes AS, Oliveira ES. Associações entre atividade física, índice de massa corporal e comportamentos sedentários em adolescentes. Rev Bras Epidemiol 2008;11:159-68.

22. Bracco MM, Colugnat FA, Pratt M, Taddei JA. Multivariate hierarchical model for physical inactivity among public school children. J Pediatr (Rio J) 2006;82:302-7.

23. American Academy of Pediatrics, Committee on Public Education. Children, adolescents, and television. Pediatrics 2001:107:423-6.

24. Patrick K, Norman GJ, Calfas KJ, Sallis JF, Zabinski MF, Rupp J et al. Diet, physical activity, and sedentary behaviors as risk factors for overweight in adolescence. Arch Pediatr Adolesc Med 2004;158:385-90.

25. Lima SC, Arrais RF, Pedrosa LF. Avaliação da dieta habitual de crianças e adolescentes com sobrepeso e obesidade. Rev Nutr 2004;17:469-77.

26. Sallis JF, Prochaska JJ, Taylor WC. A review of correlates of physical activity of children and adolescents. Med Sci Sports Exerc 2000;32:963-75.

27. Wake M, Hesketh K, Waters E. Television, computer use and body mass index in Australian primary school children. J Paediatr Child Health 2003;39:130-4.

28. Moraes SA, Rosas JB, Mondini L, Freitas IC. Prevalence of overweight and obesity, and associated factors in school children from urban area in Chilpancingo, Guerrero, Mexico, 2004. Cad Saude Publica 2006;22:1289-301.

29. Boynton-Jarrett R, Thomas TN, Peterson KE, Wiecha J, Sobol AM, Gortmaker $\mathrm{SL}$. Impact of television viewing patterns on fruit and vegetable consumption among adolescents. Pediatrics 2003;112:1321-6.

30. Wong ND, Hei TK, Qaqundah PY, Davidson DM, Bassin SL, Gold KV. Television viewing and pediatric hypercholesterolemia. Pediatrics 1992;90:75-9.

31. Malina RM, Katzmarzyk PT. Validity of the body mass index as an indicator of the risk and presence of overweight in adolescents. Am J Clin Nutr 1999;70:131S-6S 